\title{
Noradrenergic Modulation of Emotion-Induced Forgetting and Remembering
}

\author{
René Hurlemann, ${ }^{1}$ Barbara Hawellek, ${ }^{1}$ Andreas Matusch, ${ }^{3}$ Heike Kolsch, ${ }^{1}$ Heike Wollersen, ${ }^{2}$ Burkhard Madea, ${ }^{2}$ \\ Kai Vogeley, ${ }^{1}$ Wolfgang Maier, ${ }^{1}$ and Raymond J. Dolan ${ }^{4}$ \\ ${ }^{1}$ Department of Psychiatry, University of Bonn, 53105 Bonn, Germany, ${ }^{2}$ Institute of Legal Medicine, University of Bonn, 53111 Bonn, Germany, ${ }^{3}$ Institute of \\ Medicine, Research Center Juelich, 52425 Juelich, Germany, and ${ }^{4}$ Wellcome Department of Imaging Neuroscience, Institute of Neurology, University \\ College London, London WC1N 3BG, United Kingdom
}

We used a free-recall paradigm to establish a behavioral index of the retrograde and anterograde interference of emotion with episodic memory encoding. In two experiments involving 78 subjects, we show that negatively valenced items elicit retrograde amnesia, whereas positively valenced items elicit retrograde hypermnesia. These data indicate item valence is critical in determining retrograde amnesia and retrograde hypermnesia. In contrast, we show that item arousal induces an anterograde amnesic effect, consistent with the idea that a valence-evoked arousal mechanism compromises anterograde episodic encoding. Randomized double-blind administration of the $\beta$-adrenoceptor antagonist propranolol compared with the selective norepinephrine (NE) reuptake-inhibitor reboxetine, and placebo, demonstrated that the magnitude of this emotional amnesia and hypermnesia can be upregulated and downregulated as a function of emotional arousal and central NE signaling. We conclude that a differential processing of emotional arousal and valence influences how the brain remembers and forgets.

Key words: emotion; episodic memory; free recall; norepinephrine; propranolol; reboxetine

\section{Introduction}

Emotionality is often characterized along quantitative and qualitative dimensions corresponding to arousal (intensity) and valence (negative vs positive) (Bradley and Lang, 2000). Within the domains of human gustation (Small et al., 2003) and olfaction (Anderson et al., 2003), dissociable neural substrates of these dimensions have been reported, with the amygdala coding for intensity and the orbitofrontal cortex coding for valence. Accumulating evidence suggests a parallel segregation within episodic memory (Dolcos et al., 2004b; Kensinger, 2004; Kensinger and Corkin, 2004).

The initial stage of episodic memory formation is the encoding of novel stimuli (Tulving et al., 1996). It has been suggested that the influence of emotion on encoding may reflect an amygdala-mediated biasing of sensory processing and selective attention (Dolan, 2002) that prioritizes encoding of emotionally salient events (Phelps, 2004). In this context, it is interesting that an amygdala-dependent enhancement of episodic encoding has been shown to involve the central norepinephrine (NE) system (Strange et al., 2003; Strange and Dolan, 2004; van Stegeren et al., 2005). The source of central NE is the locus ceruleus (LC), which responds to novelty (Sara et al., 1994) and emotional arousal

\footnotetext{
Received Jan. 17, 2005; revised May 29, 2005; accepted May 29, 2005.

R.H. was supported by BONFOR. We thank P. Bangert, R. Fimmers, C. Frahnert, D. Schlich, D. K. Troll, and M. Wagner. In memoriam Dieter Hurlemann (1941-2005).

Correspondence should be addressed to Dr. René Hurlemann, Department of Psychiatry, University of Bonn, Sigmund-Freud-Strasse 25, 53105 Bonn, Germany. E-mail: Rene.Hurlemann@ukb.uni-bonn.de or renehurlemann@gmx.de.

DOI:10.1523/JNEUROSCI.0228-05.2005

Copyright $\odot 2005$ Society for Neuroscience $\quad$ 0270-6474/05/256343-07\$15.00/0
}

(Berridge and Waterhouse, 2003). Pharmacological manipulations that enhance a central NE response to emotional arousal (e.g., by application of reboxetine) (Harmer et al., 2003, 2004) (but see Papps et al., 2002) or the $\alpha 2$ adrenergic antagonist yohimbine (O'Carroll et al., 1999a; Southwick et al., 2002), have been shown to facilitate episodic memory formation (but see Murchison et al., 2004). In contrast, propranolol selectively compromises the memory benefit for emotionally arousing events (Cahill et al., 1994; Strange et al., 2003; van Stegeren et al., 2005) (but see O'Carroll et al., 1999b) in a manner similar to amygdala damage (Cahill et al., 1995; Strange et al., 2003). Notably, peripheral $\beta$-adrenergic blockade has no such effect (van Stegeren et al., 1998). The inconsistencies between studies we suggest are likely to reflect differing models (animals vs humans), differing test designs (modes of retrieval, delay to retrieval), and differing test material (pictorial vs verbal).

The retrograde and anterograde amnesic potential of emotional events (Tulving, 1969; Loftus and Burns, 1982; Christianson, 1984; Angelini et al., 1994) has attracted less neurobiological attention. Strange et al. (2003) showed that enhanced recall of aversive nouns is coupled to enhanced forgetting of preceding neutral nouns, with both effects eliminated by propranolol. This blockade was equivalent to that observed after bilateral amygdala damage in Urbach-Wiethe disease, implicating the amygdala and $\mathrm{NE}$ input from LC as critical targets for the action of propranolol (Strange et al., 2003).

Given that episodic memory formation is modulated by emotion (Strange et al., 2003), we wanted to clarify the distinct contribution of arousal and valence to the retrograde (and anterograde) modulation of episodic encoding by emotion. We devised 
a free-recall paradigm, in which enhanced encoding of an oddball stimulus embedded in a homogeneous sequence of neutral standard stimuli (i.e., the von Restorff phenomenon) (von Restorff, 1933; Wallace, 1965) should interfere with encoding of immediately preceding (and following) stimuli, particularly if the oddball was emotional. Subjects were instructed to encode eight stimuli from 36 von Restorff sequences, each followed by an arithmetic distracter task and an episodic memory free-recall test. In a purely behavioral experiment (experiment 1), we first established an index of the retrograde and anterograde effects of emotion. In a related psychopharmacological placebo-controlled experiment using this paradigm (experiment 2), we both blocked and enhanced a central NE response by propranolol and reboxetine, respectively, to characterize NE effects.

\section{Materials and Methods}

Subjects. Seventy-eight right-handed volunteers (39 men and 39 women) provided written informed consent to the study, which was approved by the local ethics committee and the Federal Institute for Drugs and Medical Devices (Bonn, Germany). Subjects were determined to be free of either current or past DSM-IV (Diagnostic and Statistical Manual of Mental Disorders IV) axis I disorder. They had no current physical illness and had not been on medication for $\geq 3$ months. The premenstrual week was avoided for testing female volunteers. Psychological screening before study enrollment included the Rey-Osterrieth complex figure test, a vocabulary lexical decision task, and a letter cancellation task, which showed normal to above-normal performance (data not shown).

Stimuli. The paradigm featured two categories of stimuli, oddball and standard stimuli, that consisted of a picture item and a verbal descriptor (semantically identical noun with three to seven letters; presented in arial font) to standardize stimulus verbalization at retrieval. Standard stimuli included picture items selected from a set of homogeneous black-andwhite line drawings depicting living and nonliving entities (Snodgrass and Vanderwart, 1980; Cycowicz et al., 1997). Oddball stimuli included picture items selected from the International Affective Picture System (IAPS) (Lang et al., 1997) based on their standard normative scores for emotional arousal and valence as well as additional ratings from 12 independent judges (six females; age range, 21.7-26.3 years; mean age, $23.4 \pm 1.6$ years) on a 9-point scale for arousal (1, calm; 9, excited), valence ( 1 , negative; 9 , positive) and semantic congruency of the picture and verbal component (1, lowest; 9 , highest). To equate the emotional and neutral oddballs for visual complexity and content (e.g., human presence) (Yamasaki et al., 2002), the IAPS pictures were supplemented with neutral pictures from other sources.

Of 36 oddballs presented, 12 were perceptual-neutral $(P)$, and 24 were perceptual-emotional $\left(E_{x} P\right)$, including 12 positive $\left(E_{\text {pos }} P\right)$ and 12 negative $\left(E_{\text {neg }} P\right)$ oddballs. The arousal and valence scores $($ mean $\pm \mathrm{SD})$ were as follows: $E_{\text {pos }} P$ oddballs $(5.08 \pm 0.52 ; 7.92 \pm 0.79), E_{\text {neg }} P$ oddballs $(5.33 \pm 0.65 ; 2.33 \pm 0.49)$, and $P$ oddballs $(2.92 \pm 0.67 ; 5.25 \pm 0.75)$. Only stimuli yielding high semantic congruency (range, 8-9; mean, 8.44) were implemented. Thus, $E_{\text {pos }} P$ and $E_{\text {neg }} P$ oddballs differed from each other in terms of valence but were equally arousing. $E_{x} P$ oddballs differed from $P$ oddballs in terms of valence and arousal. This was confirmed by one-factor within-subjects ANOVAs, yielding significant effects of arousal $\left(F_{(2,33)}=87.214 ; p<0.0001\right)$ and valence $\left(F_{(2,33)}=216.977 ; p<\right.$ $0.0001)$. Post hoc two-tailed one-sample $t$ tests demonstrated significant differences between (1) the arousal scores for $E_{x} P$ and $P$ oddballs (values of $p<0.05$ ), but not for $E_{\text {pos }} P$ and $E_{\text {neg }} P$ oddballs ( $p>0.05$ ), and (2) the valence scores of the three oddball types (values of $p<0.05$ ). Hence, arousal-related interference with episodic encoding should be equally induced by $E_{\text {pos }} P$ and $E_{\text {neg }} P$ oddballs, whereas differences between them should reflect valence-related interference alone.

To control for interstimulus association (cohesion), the pool of 288 stimuli was divided into 18 semantic categories (e.g., "traffic") of 16 stimuli each; 36 pseudorandom sequences composed of 8 semantically different stimuli were created, such that each sequence contained 7 standard stimuli and 1 oddball stimulus inserted on list position 3, 4, 5 or 6 to produce the von Restorff effect (von Restorff, 1933; Wallace, 1965). Serial position of oddball stimuli was balanced to reduce primacy and recency effects (i.e., the enhanced probability for the first few and the last few stimuli of a sequence to be recalled better) (Capitani et al., 1992).

As indicated by pilot data (data not shown), the restriction to 8 items per input sequence was effective in balancing out a lower recall of $P$ oddballs and a higher recall of $E_{x} P$ oddballs reported for lists of 15 items (Strange et al., 2003). This equation of von Restorff distinctiveness across oddball types allowed us to remove a potential confounding effect of differential distinctiveness on recall of items immediately neighboring oddball presentation. Consequently, the influence of positive and negative emotion could be isolated and quantified.

Experiment 1. Twenty-four subjects (12 females; age range, 21.3-28.2 years; mean age, $24.6 \pm 1.9$ years) had visual stimuli presented sequentially at a rate of one every $5 \mathrm{~s}$ (stimulus duration, $2 \mathrm{~s}$; followed by a $3 \mathrm{~s}$ fixation cross) in the center of a 17 inch flat screen. During each of three runs (duration, $20 \mathrm{~min} ; 3$ min breaks between runs), volunteers were presented with 12 input sequences (4 sequences per oddball type, randomly assigned), introduced by presentation of the words "New Sequence." Subjects engaged in a deep encoding task (dichotomous "natural vs artificial" judgment for each stimulus, indicated by push-button response). This was immediately followed by a working memory task in form of an arithmetic distracter task, announced by a triple-digit number (e.g., 173) presented for $30 \mathrm{~s}$, from which subjects were required to count back serially in sevens. Such interpolation of a filled delay between study and test served to eliminate recency effects in free recall (Capitani et al., 1992). Furthermore, ongoing rehearsal of list items was prevented. Episodic memory was subsequently tested by free recall. The words "Please Remember" were displayed for $30 \mathrm{~s}$, requesting subjects to spontaneously recall as many pictures, using their verbal descriptors, as they could from the just-seen input list before continuing with the next input list. Figure $1 A$ illustrates the experimental timeline.

The recorded free-recall profiles were pooled according to the three oddball types, consequently yielding a positive $\left(E_{\text {pos }} P\right)$, negative $\left(E_{\text {neg }} P\right)$, and neutral (control) $(P)$ condition. As outcome parameter, memory performance was determined for each condition by calculating the percentage of correct recall (i.e., the output/input ratio) for the following three list positions: oddball -1 , oddball, and oddball +1 . Additionally, a sequence index (SI) based on the seven non-oddball list positions was calculated for each condition (e.g., $\mathrm{SI}_{P}$ ). Contrasting the emotional conditions with the neutral condition (according to $E_{x} P-P=E_{x}$ ) allowed us to specify retrograde and anterograde effects produced by positive $\left(E_{\text {pos }}\right)$ and negative $\left(E_{\text {neg }}\right)$ emotion on one adjacent standard event $\left(E_{x} \pm\right.$ $1)$ corresponding to a time window of $\pm 5 \mathrm{~s}$ (see Fig. $1 B$ ).

Experiment 2. Fifty-four subjects (27 females; age range, 20.6-29.5 years; mean age, $26.2 \pm 2.2$ years) were randomly allocated to doubleblind oral intake of one of the three following capsules: lactose placebo, a $40 \mathrm{mg}$ single dose of the $\beta$-adrenoceptor antagonist propranolol hydrochloride, or a $4 \mathrm{mg}$ single dose of the selective NE reuptake-inhibitor reboxetine mesilate. Drug allocation was balanced for gender [i.e., nine males and nine females were randomly assigned to each group $(n=18)]$. In view of the different kinetics (time to peak plasma concentration, propranolol, 1-2 h; elimination half-life, 3-4 h; reboxetine, 2 and $13 \mathrm{~h}$, respectively), the time interval between ingestion of the capsule and start of the memory task was 90 min (propranolol group and one-half of the placebo group; sample A) and $120 \mathrm{~min}$ (reboxetine group and the other one-half of the placebo group; sample B), respectively. The memory task (identical to experiment 1) started at $t=0 \mathrm{~min}$ and finished at $t=66$ min. Blood pressure and pulse frequency were monitored according to the following timelines: sample A, $-90,-60,-30,0,20,43$, and $66 \mathrm{~min}$; sample $\mathrm{B},-120,-90,-60,-30,0,20,43$, and $66 \mathrm{~min}$. At $t=70 \mathrm{~min}$, a venous blood sample was taken to determine drug plasma concentrations. Afterward, subjects performed computerized valence and arousal ratings for each oddball stimulus to assess potential drug effects on emotional experience. Note that the experimenter was blinded for the type of pharmacological treatment.

As an emotion-induced retrograde amnesia is $\beta$-adrenergic dependent (Strange et al., 2003), our prediction for experiment 2 was that $\beta$-adrenergic blockade with propranolol should abolish potential $E_{x} \pm 1$ 
effects evoked by positive $\left(E_{\text {pos }}\right)$ and negative $\left(E_{\text {neg }}\right)$ emotion, whereas NE reuptake inhibition with reboxetine should produce a converse pattern. Recall parameters (identical with those of experiment 1) were calculated group-wise for statistical analysis. In a first step, data obtained from experiment 1 were compared with the placebo group in experiment 2 to verify that $E_{x} \pm 1$ effects were not affected by placebo administration or other procedural differences. In a second step, the emotional conditions for propranolol and reboxetine groups were contrasted with the placebo neutral condition (neutral baseline) to index drug-related changes of $E_{x} \pm 1$ effects.

Plasma drug levels were analyzed by HPLC. Propranolol plasma samples were extracted with dichlormethane at $\mathrm{pH}$ 9. After evaporation, 100 $\mu \mathrm{l}$ of mobile phase was added to the residue and injected on a LichroCart 250-4, RP-8, $5 \mu \mathrm{m}$ column (Merck, Darmstadt, Germany). The analyte was isocratically eluted with acetonitrile-potassium phosphate buffer $(50: 50 ; \mathrm{v} / \mathrm{v})$ as mobile phase. Propranolol was detected by ultraviolet (UV) absorption at $210 \mathrm{~nm}$. The absolute extraction recovery was $>85 \%$. Reboxetine plasma samples first underwent solid-phase extraction as described by Frahnert et al. (2003). Chromatographic separation of reboxetine was achieved on a Nucleosil 100-Protect 1 column (MachereyNagel, Dueren, Germany) with acetonitrile-potassium phosphate buffer $(40 / 60 ; \mathrm{v} / \mathrm{v})$ as mobile phase. Reboxetine was detected by UV absorption at $230 \mathrm{~nm}$. The absolute extraction recovery was $>95 \%$.

Statistics. Recall parameters $\left(E_{x}, E_{x} \pm 1, \mathrm{SI}_{X}\right)$ of the emotional (positive, negative) conditions were analyzed in relation to the corresponding recall parameters $\left(P, P \pm 1, \mathrm{SI}_{P}\right)$ of the neutral (control) condition. Two-factor within-subjects and three-factor mixed ANOVAs were followed by two-tailed one-sample and two-sample $t$ tests to determine the source of significance. Greenhouse-Geisser correction for inhomogeneity of variance was applied whenever a sphericity assumption was violated. To account for an inflation of the type I error rate attributable to multiple post hoc testing, the threshold for significance was Bonferroni adjusted.

\section{Results}

\section{Experiment 1}

The aim of experiment 1 was to determine emotion-induced effects on recall of $E_{x} \pm 1$ stimuli (Fig. $1 B$ ). The percentage (\%) of mean recall $( \pm \mathrm{SD})$ collapsed across conditions was $95.37(6.84)$ for oddball stimuli and 57.59 (10.09) for standard stimuli, demonstrating a near-ceiling von Restorff effect (one-sample $t$ test; $t_{(23)}=-19.535 ; p<0.0001$ ) (Fig. 1Ci). A condition (positive, negative, neutral) $\times$ position (oddball, oddball \pm 1$) 3 \times 3$ ANOVA yielded significant condition $\left(F_{(2,46)}=14.010 ; p<\right.$ $0.0001)$, position $\left(F_{(1.6,36.2)}=342.070 ; p<0.0001\right)$, and condition $\times$ position interaction $\left(F_{(4,92)}=16.150 ; p<0.0001\right)$ effects. Post hoc one-sample $t$ tests demonstrated that $E_{\text {neg }}-1$ events were recalled less well $(-11.46 \%)$ than $P-1$ events $\left(t_{(23)}=\right.$ $-3.058 ; p=0.006)$, whereas $E_{\text {pos }}-1$ events were recalled better $(+12.85 \%)$ than $P-1$ events $\left(t_{(23)}=4.270 ; p<0.0001\right)$. In addition, an $E_{\text {neg }}+1$ decrement of $11.81 \%\left(t_{(23)}=-3.593 ; p=\right.$ $0.002)$ and an $E_{\text {pos }}+1$ decrement of $9.38 \%\left(t_{(23)}=-3.452 ; p=\right.$ 0.002 ) were observed (Fig. 1Cii). There was no influence of condition on oddball recall (values of $p>0.05$ ), confirming the presence of equivalent von Restorff effects across conditions. Thus, positive and negative emotion produced dissociable $E_{x}-1$ effects, implying a differential modulation of $E_{x}-1$ postexposure processing as a function of emotional valence. Figure $1, C i$ and Cii, illustrates the results of experiment 1.

An additional gender (male, female) $\times$ emotion (negative, positive $) \times$ position $2 \times 2 \times 3$ ANOVA detected no significant influence of gender $\left(F_{(1,22)}=1.028 ; p>0.05\right)$ (i.e., the amnesic and hypermnesic effects were equally strong in male and female volunteers). Run $\times$ condition $3 \times 3$ within-subjects ANOVAs revealed that memory performance for oddballs $\left(F_{(2,142)}=2.298\right.$; $p>0.05)$ and standard stimuli $\left(F_{(2,142)}=1.062 ; p>0.05\right)$ re-
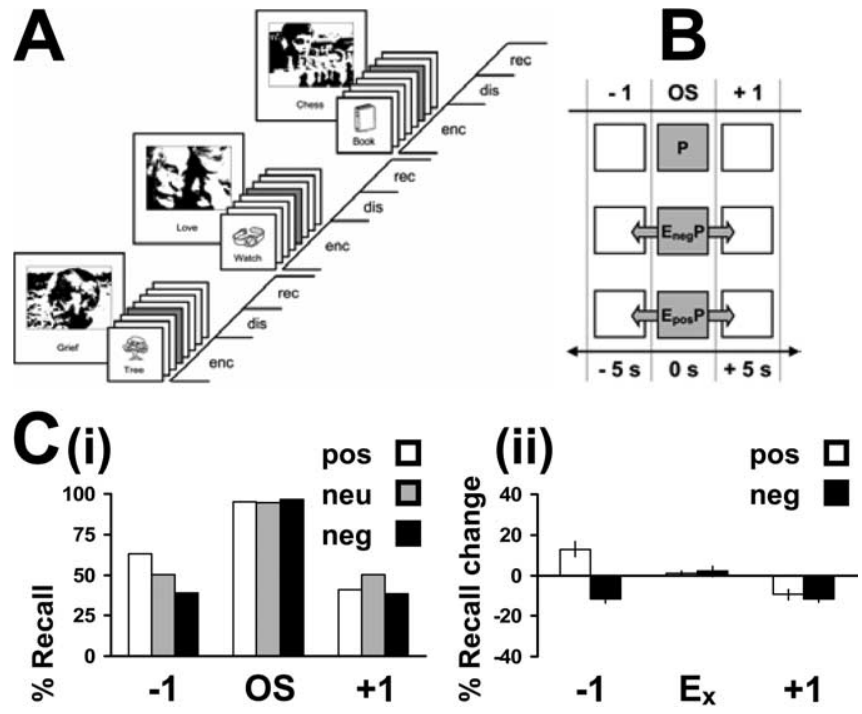

Figure 1. $A$, Experimental timeline. Subjects were instructed to encode eight stimuli from 36 input lists (enc) (40 s), each followed by a distracter task (dis) (30 s) and a free-recall test to probe episodic memory (rec) (30 s). $\boldsymbol{B}$, Experimental design. In each input list, one oddball stimulus $(0 S)$, either emotionally salient $\left(E_{x} P\right.$; positive, $E_{\text {pos }} P$; negative, $\left.E_{\text {neg }} P\right)$ or neutral $(P)$, was interspersed on position $3,4,5$, or 6 , such that it was temporally flanked by two or more standard stimuli. Results from list recall were pooled, thus yielding a positive (pos), negative (neg), and neutral (neu) condition. Contrasting the emotional conditions with the neutral condition (according to $E_{X} P-P=E_{x}$ ) allowed us to extract retrograde and anterograde effects of positive and negative emotion on episodic memory encoding within a time window of $E_{x} \pm 1$ stimuli or $\pm 5 \mathrm{~s}$. Ci, Percentage (\%) recall in experiment $1(n=24)$. Equal (near-ceiling) von Restorff effects were obtained for emotional and neutral oddballs. In contrast, memory for immediately preceding $\left(E_{x}-1\right)$ but not for following $\left(E_{x}+1\right)$ items depended on oddball valence. Cii, Recall change $(\%)$ relative to the neutral condition. Whereas negative emotion $\left(E_{\text {neg }}\right)$ elicited $E_{\text {neg }}-1$ retrograde amnesia $(-11.46 \%)$, positive emotion $\left(E_{\text {pos }}\right)$ evoked $E_{\text {pos }}-$ 1 retrograde hypermnesia $(+12.85 \%)$. An $E_{x}+1$ amnesic effect followed both $E_{\text {neg }}$ $(-11.81 \%)$ and $E_{\text {pos }}(-9.38 \%)$. Error bars indicate SE.

mained unchanged throughout the experiment (i.e., neither practice nor adaptation effects occurred in any condition).

\section{Experiment 2}

Free recall

The focus of the psychopharmacological experiment was to manipulate $E_{x} \pm 1$ effects by double-blind placebo-controlled modulation of central NE using the $\beta$-adrenoceptor antagonist propranolol and the selective NE reuptake-inhibitor reboxetine. A procedure (no intake in experiment 1 , placebo intake in experiment 2) $\times$ condition (positive, negative, neutral) $\times$ position (oddball, oddball \pm 1 ) $2 \times 3 \times 3$ ANOVA confirmed that placebo administration per se did not affect recall performance in comparison with experiment $1\left(F_{(1,40)}=0.043 ; p>0.05\right)$. Moreover, no significant interactions with the between-subjects factor were found. A condition $\times$ position $3 \times 3$ ANOVA restricted to the placebo group yielded effects of valence $\left(F_{(2,34)}=14.000 ; p<\right.$ $0.0001)$ and position $\left(F_{(2,34)}=241.725 ; p<0.0001\right)$, and a significant interaction of these factors $\left(F_{(2.5,41.7)}=12.936 ; p<\right.$ $0.0001)$. As depicted in Figure 2, Ai and Bi, post hoc one-sample $t$ tests verified the presence of $E_{\text {neg }}-1$ amnesic $(-14.82 \%)\left(t_{(17)}=\right.$ $-4.531 ; p<0.0001), E_{\text {pos }}-1$ hypermnesic $(+15.74 \%)\left(t_{(17)}=\right.$ $3.213 ; p=0.005)$ as well as $E_{\text {neg }}+1(-14.35 \%)\left(t_{(17)}=-3.019\right.$; $p=0.008)$ and $E_{\mathrm{pos}}+1(-12.96 \%)\left(t_{(17)}=-3.197 ; p=0.005\right)$ amnesic effects in the placebo group. Hence, placebo group findings replicated those obtained in experiment 1 and served as an appropriate baseline for subsequent comparisons.

A one-way ANOVA with group (placebo, propranolol, rebox- 
etine) as between-subjects factor showed that $\mathrm{SI}_{P}$ scores were unaffected by drug treatment $\left(F_{(2,51)}=0.394 ; p>0.05\right)$ [i.e., neither propranolol nor reboxetine administration altered recall performance in the corresponding neutral (nontarget) conditions]. Analyzing the influence of propranolol, a group (placebo, propranolol) $\times$ condition $\times$ position $2 \times 3 \times 3$ ANOVA yielded condition $\left(F_{(2,68)}=\right.$ $6.501 ; p=0.003)$, position $\left(F_{(2,68)}=\right.$ 428.481; $p<0.0001)$, and three-way group $\times$ condition $\times$ position interaction $\left(F_{(2.9,99.6)}=6.288 ; p=0.001\right)$ effects. A subsequent $2 \times 2 \times 3$ ANOVA restricted to the emotional factor revealed group $\left(F_{(1,34)}=5.127 ; p=0.030\right)$, condition $\left(F_{(1,34)}=10.754 ; p=0.002\right)$, position $\left(F_{(2,68)}=494.071 ; p<0.0001\right)$, and threeway interaction $\left(F_{(1.6,52.7)}=7.798 ; p=\right.$ $0.002)$ effects. Post hoc two-sample $t$ tests showed that propranolol abolished the $E_{\text {pos }}-1$ recall increment $\left(t_{(34)}=2.919\right.$; $p=0.006)$ as it lessened $E_{\text {neg }}-1\left(t_{(34)}=\right.$ $-3.238 ; p=0.003), E_{\text {neg }}+1\left(t_{(34)}=\right.$ $-2.796 ; p=0.008)$, and $E_{\mathrm{pos}}+1\left(t_{(34)}=\right.$ $-2.836 ; p=0.008)$ recall decrements. No changes in oddball recall were observed (values of $p>0.05$ ). Figure 2, Aii and Bii, illustrate that propranolol attenuated $E_{x} \pm$ 1 recall to the level of $P \pm 1$ recall.

Analyzing the influence of reboxetine, a $2 \times 3 \times 3$ ANOVA yielded group $\left(F_{(1,34)}=\right.$ 4.930; $p=0.033)$, condition $\left(F_{(2,68)}=\right.$ $52.251 ; p<0.0001)$, position $\left(F_{(2,68)}=\right.$ 481.260; $p<0.0001$ ), and three-way group $\times$ condition $\times$ position interaction $\left(F_{(3.2,109.3)}=5.226 ; p<0.0001\right)$ effects. Post hoc two-sample $t$ tests indicated a significant effect on $E_{x}-1$ recall (enhancement of $E_{\text {neg }}$-induced amnesia and $E_{\text {pos }}$-induced hypermnesia). There was an overall valence-independent enhancement of $E_{x}+1$ effects relative to the placebo group, which reached uncorrected significance, but did not survive Bonferroni correction. The percentage $(\%)$ recall changes relative to the corresponding $E_{x} \pm 1$ scores (relative to the $P \pm 1$ scores) of the placebo group were as follows: $E_{\text {neg }}-1,-19.91(-34.72)\left(t_{(34)}=4.690 ; p<0.0001\right)$; $E_{\text {pos }}-1,+14.82(+30.56)\left(t_{(34)}=-2.931 ; p=0.006\right) ; E_{\text {neg }}+1$, $-12.96(-27.32)\left(t_{(34)}=2.633 ; p=0.013\right) ; E_{\text {pos }}+1,-12.50$ $(-25.46)\left(t_{(34)}=2.715 ; p=0.010\right)$. No changes in oddball recall were present (values of $p>0.05$ ) (Fig. 2Aiii,Biii).

Comparing $E_{\text {neg }}-1$ recall with $E_{\text {pos }}-1$ recall indicated equal strength of reboxetine-induced elevations of $E_{x}-1$ amnesia and hypermnesia (one-sample $t$ test; $t_{(17)}=0.823 ; p>0.05$ ). In summary, propranolol administration attenuated the retrograde and anterograde effects of emotion, whereas NE reuptake-inhibition with reboxetine enhanced retrograde interference more significantly than anterograde interference.

Two ANOVAs with group as between-subjects factor confirmed propranolol effects on systolic $\left(F_{(1,34)}=18.502 ; p<\right.$ $0.0001)$ and diastolic $\left(F_{(1,30)}=11.368 ; p<0.0001\right)$ blood pressure at the start of the cognitive task. Reboxetine had no such effects (values of $p>0.05$ ). As illustrated in Figure 2, Ci-Ciii, both systolic $\left(t_{(34)}=4.826 ; p<0.0001\right)$ and diastolic $\left(t_{(34)}=\right.$
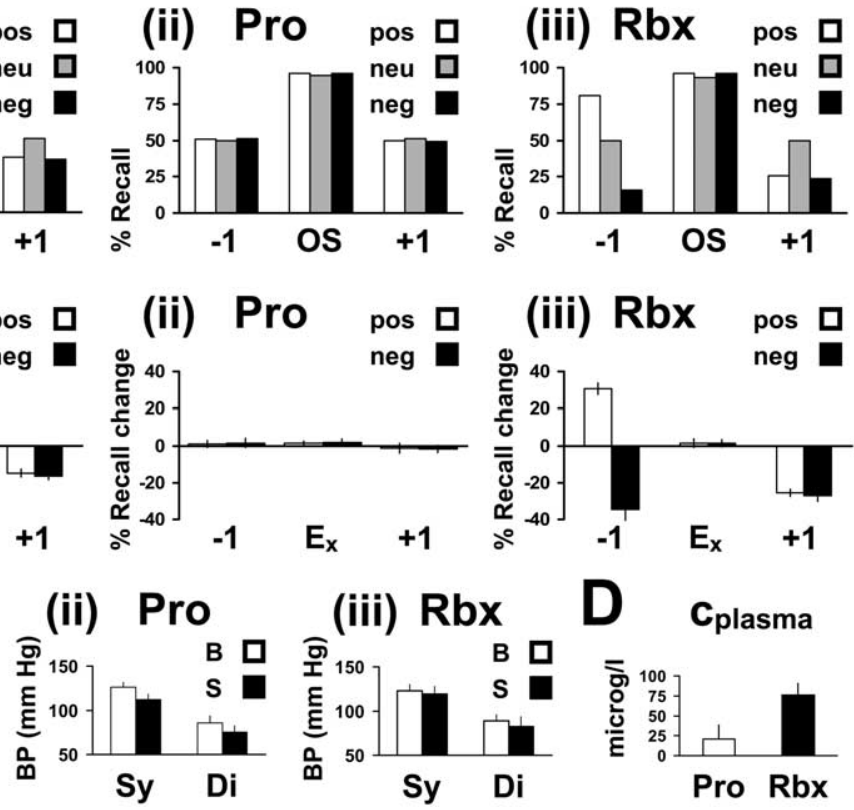

Figure 2. $\boldsymbol{A i}$, Percentage (\%) recall in experiment 2, placebo group $(n=18)$. Aii, Percentage $(\%)$ recall in experiment 2 , propranolol group $(n=18)$. Aiii, Percentage (\%) recall in experiment 2, reboxetine group $(n=18)$. Independent of treatment, (n) von Restorff effects were present for emotional and neutral oddball stimuli (OS). Note that none of the drugs ( millimeters mercury), at the time of drug/placebo administration (B) and at the start of the cognitive task (S). Wereas BP was unaffected by reboxetine, propranolol produced the expected BP decrease. $D$, Propranolol and reboxetine plasma concentrations $\left(C_{\text {plasma }}\right.$ ) (micrograms/liter). $E_{x^{\prime}}$ Emotion contact; $E_{x} \pm 1, \pm 1$ stimulus (or $5 \mathrm{~s}$ ) adjacent to $E_{x}$. Error bars indicate $S E$. Plac, Placebo; Pro, propranolol; Rbx, reboxetine.

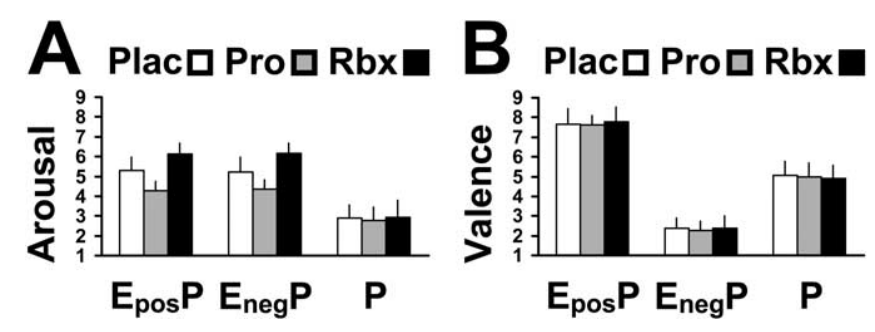

Figure 3. Drug-related effects on oddball ratings compared with placebo (Plac). $\boldsymbol{A}$, Arousal judgments. Propranolol (Pro) decreased arousal scores for negative $\left(E_{\text {neg }} P\right)$ and positive $\left(E_{\text {pos }} P\right)$ oddball stimuli, whereas reboxetine $(\mathrm{Rbx})$ increased $E_{\text {neg }} P$ and $E_{\text {pos }} P$ arousal scores, thus indicating a noradrenergic modulation of arousability. $\boldsymbol{B}$, Valence judgments. No drug-related effects on valence scores were observed. Error bars indicate SE.

3.842; $p=0.001$ ) blood pressure were decreased by propranolol ( post hoc two-sample $t$ tests). Figure $2 D$ depicts the plasma concentrations of both propranolol $(20.50 \pm 17.96 \mu \mathrm{g} / \mathrm{L})$ and reboxetine $(75.11 \pm 15.79 \mu \mathrm{g} / \mathrm{L})$ as measured after the recall task.

\section{Oddball ratings}

Figure 3 illustrates the arousal (Fig. $3 A$ ) and valence (Fig. $3 B$ ) scores (mean $\pm \mathrm{SD}$ ) obtained after memory testing. For placebo treatment, scores were as follows: $E_{\text {pos }} P$ oddballs (5.28 \pm 0.67 ; $7.67 \pm 0.77), E_{\text {neg }} P$ oddballs $(5.22 \pm 0.73 ; 2.39 \pm 0.50)$, and $P$ oddballs $(2.88 \pm 0.68 ; 5.06 \pm 0.73)$. For propranolol treatment, scores were as follows: $E_{\text {pos }} P$ oddballs ( $\left.4.28 \pm 0.46 ; 7.61 \pm 0.50\right)$, 
$E_{\text {neg }} P$ oddballs $(4.33 \pm 0.49 ; 2.28 \pm 0.46), P$ oddballs $(2.78 \pm$ $0.65 ; 5.00 \pm 0.69)$. For reboxetine treatment, scores were as follows: $E_{\text {pos }} P$ oddballs $(6.11 \pm 0.58 ; 7.78 \pm 0.73), E_{\text {neg }} P$ oddballs (6.17 $\pm 0.52 ; 2.39 \pm 0.61)$, and $P$ oddballs ( $2.94 \pm 0.87 ; 4.89 \pm$ 0.68 ). Separate treatment (placebo, drug) $\times$ oddball type (positive, negative, neutral) $2 \times 3$ ANOVAs were calculated, yielding significant effects of propranolol $\left(F_{(1,34)}=65.964 ; p<0.0001\right)$ and reboxetine $\left(F_{(1,34)}=27.508 ; p<0.0001\right)$ on arousal scores. In another series of ANOVAs, no group effects on valence scores were found (values of $p>0.05$ ). Post hoc two-sample $t$ tests demonstrated significant decreases of arousal scores for emotional oddballs, but not for neutral oddballs, under propranolol: $E_{\text {pos }} P$ oddballs $\left(t_{(34)}=5.222 ; p<0.0001\right), E_{\text {neg }} P$ oddballs $\left(t_{(34)}=\right.$ 4.294; $p<0.0001)$, and $P$ oddballs $\left(t_{(34)}=0.504 ; p>0.5\right)$. In contrast, a significant increase of arousal scores for emotional oddballs, but not for neutral oddballs, was observed under reboxetine: $E_{\text {pos }} P$ oddballs $\left(t_{(34)}=-3.984 ; p<0.0001\right), E_{\text {neg }} P$ oddballs $\left.t_{(34)}=-4.478 ; p<0.0001\right)$, and $P$ oddballs $\left(t_{(34)}=-0.213 ; p>\right.$ $0.5)$. These results provide substantial evidence for a pharmacological modulation of emotional stimulus-evoked arousal, suggesting that propranolol decreased subjects' arousability, whereas reboxetine increased subjects' arousability.

\section{Discussion}

Our data demonstrate distinct functional roles for valence and arousal in the retrograde modulation of episodic memory by emotion. We emphasize that the effects we observed pertain to those that operate over short time scales, and it would be of interest to examine these effects over a longer time scale. The quality of retrograde effects (i.e., $E_{\text {neg }}$-induced extinction vs $E_{\text {pos }}$ induced facilitation of $E_{x}-1$ events) is determined by emotional valence. In contrast, the quantitative aspects of retrograde effects (i.e., the magnitude of $E_{x}-1$ amnesia and hypermnesia) may depend on emotional arousal. By pharmacologically manipulating the central NE system using a double-blind placebocontrolled experimental design, we show that the $\beta$-adrenoceptor antagonist propranolol decreased arousal and blocked $E_{x}-1$ effects, whereas the selective NE reuptakeinhibitor reboxetine increased subjects' arousal and enhanced valence-specific $E_{x}-1$ effects.

In humans, few studies have examined NE effects on memory (Gold and van Buskirk, 1978) by challenging the central NE system pharmacologically. Whereas yohimbine-elicited NE potentiation amplified consolidation and free recall of emotional events (O'Carroll et al., 1999a; Southwick et al., 2002), no effect of prelearning single-dose administrations of 4 and $8 \mathrm{mg}$ reboxetine on emotional recall tested after a $7 \mathrm{~d}$ retention interval has also been reported (Papps et al., 2002). In contrast, Harmer et al. (2003) found that a $4 \mathrm{mg}$ single dose of reboxetine administered $2 \mathrm{~h}$ before testing enhanced free recall of positive rather than negative stimuli in the absence of changes in nonemotional performance or mood. The bias of reboxetine for recall of positive stimuli (Harmer et al., 2003, 2004) is interesting insofar as the $E_{\text {pos }}$-induced retrograde hypermnesia we report might depend on a similar mechanism. The underlying mechanism is valence specific in that it has opposite effects on $E_{\text {neg }}$-induced retrograde effects. Because reboxetine enhanced arousal, the evidence from our study points to a valence-arousal interaction effect. Note that our experimental design was optimized to examine modulatory effects of emotional items on encoding of temporally contiguous neutral items and not on recall of emotional items per se. We carefully equated positive and negative stimuli for emotional arousal to probe drug performance, accounting for the asymmet- rical distribution of arousal between valences (Anderson et al., 2003) and its potential biasing of pharmacological effects. Whatever the underlying mechanism, the elevation by reboxetine and the blockade by propranolol of retrograde effects indicate that both $E_{\text {neg }}$-induced forgetting and $E_{\text {pos }}$-induced remembering are critically dependent on central NE signaling.

In contrast to our previous study (Strange et al., 2003), we used shorter input sequences to match oddball stimuli for von Restorff distinctiveness, resulting in near-ceiling recall of oddball stimuli. This equalization of recall performance across oddball types enabled us to specify the contribution of valence to retrograde and anterograde modulations of episodic memory encoding. However, differential enhancement of recall for oddball stimuli or more subtle effects of gender were not detectable in this paradigm, reflecting a cost of change in experimental design. Despite these methodological differences, our results are consistent with, and expand on, our previous findings (Strange et al., 2003). Of particular importance is that amnesia was not restricted to encoding of $E_{x}-1$ events, but extended to encoding of $E_{x}+1$ events, perhaps implying a higher intrinsic arousal of combined pictorial and verbal emotional stimuli compared with verbal items alone as the source of effect. This $E_{x}+1$ anterograde amnesia during mnemonic processing of serially presented stimuli implicates a mechanism based on an arousal-induced interruption of stimulus processing. One potential mechanism for this might be emotional item capture of selective attention, such that less processing resources are available for subsequent items.

The above interpretation is in keeping with findings using the "attentional blink" paradigm, in which detection of an initial target in a visual stimulus stream induces "inattentional blindness" for a successive second target (Raymond et al., 1992). Although the attentional blink is attenuated when the second target is emotionally arousing, this attenuation is abolished in patients with amygdala damage (Anderson and Phelps, 2001). Furthermore, patients with amygdala damage do not show a trade-off between memory for emotional gist and nonemotional details of complex visual stimuli (Adolphs et al., 2005). Compatible with the hypothesis of Easterbrook (1959) and the "weapon-focus" phenomenon in eyewitness testimony (Loftus, 1979), these patient data suggest an amygdala role in directing the attentional focus to emotionally arousing events, thus leaving less capacity available for processing competing stimuli.

Intra-amygdalar infusions of NE agonists and antagonists provide evidence that amygdala functions are under NE control (McGaugh, 2000). In support of these animal data, amygdala activation at encoding of emotionally arousing stimuli has been shown to depend on amygdala NE (Strange and Dolan, 2004; van Stegeren et al., 2005). Under placebo conditions, amygdala responses are enhanced to emotional stimulus-evoked arousal, suggesting that encoding strength varies as a function of amygdala engagement (van Stegeren et al., 2005). In view of NE as a key neurochemical factor in the modulation of encoding efficiency by the amygdala (Strange et al., 2003; Strange and Dolan, 2004; van Stegeren et al., 2005), the amplification by reboxetine (albeit not surviving Bonferroni correction in our study) and the attenuation by propranolol of $E_{x}+1$ effects indicate a narrowing or broadening of an access window to episodic memory as a function of amygdala NE levels. We suggest that these $E_{x}+1$ anterograde amnesic effects reflect the costs of deploying resources to preferential processing of preceding emotionally arousing events (Dolan, 2002).

Tulving (1969) conjectured that retrograde amnesia in free recall could arise from premature termination of encoding. In 
line with our previous findings (Strange et al., 2003), $E_{\text {neg }}-1$ retrograde amnesia, and the current enhancement of this effect with reboxetine, is consistent with an emotional arousal cost on ongoing episodic encoding. This premature termination is not expressed when an arousing item is positive, and indeed there is an opposite effect of hypermnesia for preceding items. This calls for a more complex explanation that invokes an interaction between arousal and valence in the expression of these retrograde effects on memory.

In experiment 2, the effects of propranolol and reboxetine spanned both encoding and free recall. Thus, we cannot rule out that pharmacological manipulations of the NE system affected retrieval (Murchison et al., 2004) rather than encoding stages of episodic memory. Separation of encoding and retrieval effects on the basis of behavioral data alone is extremely problematic. Substantial evidence links NE release during emotional arousal to an amygdala modulation of hippocampal function at encoding. Anatomical studies in rats demonstrate reciprocal amygdalohippocampal connections (Pitkanen et al., 2000). Electrical stimulation of the amygdala elicits propranolol-sensitive retrograde amnesia (Sternberg and Gold, 1981). Amygdala activation at encoding correlates with subsequent memory for emotionally arousing items (Cahill et al., 1996; Hamann et al., 1999; Canli et al., 2000; Dolcos et al., 2004a; Kensinger and Corkin, 2004). Investigation of patients with amygdalohippocampal pathology has revealed a reciprocal dependence between amygdala and hippocampus during encoding of emotionally arousing stimuli (Richardson et al., 2004). Such cross talk at encoding can be blocked by propranolol (Strange and Dolan, 2004), thus abolishing the retrieval advantage of emotional stimuli, despite propranolol being no longer active at retrieval (van Stegeren et al., 2005). Such findings favor amygdala-NE modulation of encoding efficiency (McGaugh and Roozendaal, 2002; Strange et al., 2003). One hypothesis is that the emotion-induced forgetting and remembering we observed results from functional alterations in amygdalohippocampal interactions that might be orchestrated by NE. However, the complexity of these processes needs to be acknowledged given the valence-dependent retrograde effects we observe.

Previous psychopharmacological studies have not reported an effect of drug manipulation of NE on arousal ratings (Cahill et al., 1994; van Stegeren et al., 1998, 2005; O'Carroll et al., 1999a,b; Papps et al., 2002), whereas we show such effects. The critical variable here is likely to be the paradigm we used involving presentation of pictorial and verbal descriptors of emotional items. The effects we observed suggest a link between the appraisal of emotional arousal and its physiological response, thus implicating engagement of interconnected brain areas. A logical candidate site is the prefrontal cortex, which shows an arousal-related parcellation (Dolcos et al., 2004b) and receives input from both amygdala (Barbas, 2000) and LC (Arnsten and Goldman-Rakic, 1984; Arnsten, 1997; Aston-Jones et al., 2000; Berridge and Waterhouse, 2003).

Dissociable contributions of arousal and valence to the retrograde effects of emotion correspond to a segregation of these dimensions on the neural level (Anderson et al., 2003; Small et al., 2003; Dolcos et al., 2004b; Kensinger, 2004; Kensinger and Corkin, 2004). Compatible with Kensinger and Corkin (2004), we suggest that the magnitude of the retrograde effects of emotion varies as a function of arousal perhaps under the control of amygdalohippocampal connectivity, whereas their quality (i.e., the occurrence of retrograde amnesia vs hypermnesia) may be related to a valence-dependent activation of prefrontal cortex-hippocampal circuits. Support for this interpretation comes from
Dolcos et al. (2004b), who identified a valence-specific parcellation within prefrontal cortex. Thus, positively valenced arousing stimuli might engage specific subdivisions of prefrontal cortex to inhibit arousal-related disruption of $E_{x}-1$ encoding in hippocampus, thus augmenting ongoing encoding processes and inducing $E_{\text {pos }}-1$ hypermnesia. Hence, unlike the evolutionarily conserved role of the amygdala in mediating modulatory effects of emotional arousal, it seems that a valence-related modulation of ongoing episodic encoding may reflect a more refined control function mediated by prefrontal cortex (for a similar proposal on perception, see Anderson et al., 2003).

Three conclusions emerge from our observations. First, the neurotransmitter NE modulates episodic encoding independently of emotional valence (Berridge and Waterhouse, 2003) (for related results on glucocorticoids, see Buchanan and Lovallo, 2001). Consistent with animal data (McGinty and Szymusiak, 1988), arousal-associated NE signaling may thus reflect a change in the intensity of emotional events. Second, prelearning application of propranolol and reboxetine interferes with NE neurotransmission allowing upregulation and downregulation of emotional arousability as well as the magnitude of emotional amnesia and hypermnesia. The effects depend on arousal but are not solely explicable in these terms in that we show differential effects as a function of valence, indicating an interaction effect between these two factors. Third, compatible with a motivational perspective on emotion (Hamann, 2001; Dolan, 2002; Wise, 2004), emotional valence appears to be critical for retrograde forgetting versus remembering. Perhaps positive emotion promotes retention of information that predicts reward (positive items), reflecting an influence of "return to the rewards, and to the cues marking the way to such rewards" (Wise, 2004). Thus, computing the positive and negative value of emotionally arousing stimuli might be a means that the brain uses to organize forgetting and remembering.

\section{References}

Adolphs R, Tranel D, Buchanan TW (2005) Amygdala damage impairs emotional memory for gist but not details of complex stimuli. Nat Neurosci 8:512-518.

Anderson AK, Phelps EA (2001) Lesions of the human amygdala impair enhanced perception of emotionally salient events. Nature 411:305-309.

Anderson AK, Christoff K, Stappen I, Panitz D, Ghahremani DG, Glover G, Gabrieli JD, Sobel N (2003) Dissociated neural representations of intensity and valence in human olfaction. Nat Neurosci 6:196-202.

Angelini R, Capozzoli F, Lepore P, Grossi D, Orsini A (1994) “Experimental amnesia" induced by emotional items. Percept Mot Skills 78:19-28.

Arnsten AF (1997) Catecholamine regulation of the prefrontal cortex. J Psychopharmacol 11:151-162.

Arnsten AF, Goldman-Rakic P (1984) Selective prefrontal cortical projections to the region of the locus coeruleus and raphe nucleus in the rhesus monkey. Brain Res 306:9-18.

Aston-Jones G, Rajkowski J, Cohen J (2000) Locus coeruleus and regulation of behavioral flexibility and attention. Prog Brain Res 126:165-182.

Barbas H (2000) Connections underlying the synthesis of cognition, memory, and emotion in primate prefrontal cortices. Brain Res Bull 2000 52:319-330.

Berridge CW, Waterhouse BD (2003) The locus coeruleus-noradrenergic system: modulation of behavioral state and state-dependent cognitive processes. Brain Res Brain Res Rev 42:33-84.

Bradley MM, Lang PJ (2000) Measuring emotion: behavior, feeling, and physiology. In: Cognitive neuroscience of emotion (Lane RD, Nadel L, eds), pp 242-277. New York: Oxford UP.

Buchanan TW, Lovallo WR (2001) Enhanced memory for emotional material following stress-level cortisol treatment in humans. Psychoneuroendocrinology 26:307-317.

Cahill L, Prins B, Weber M, McGaugh JL (1994) $\beta$-Adrenergic activation and memory for emotional events. Nature 371:702-704. 
Cahill L, Babinsky R, Markowitsch HJ, McGaugh JL (1995) The amygdala and emotional memory. Nature 377:295-296.

Cahill L, Haier RJ, Fallon J, Alkire MT, Tang C, Keator D, Wu J, McGaugh JL (1996) Amygdala activity at encoding correlated with long-term, free recall of emotional information. Proc Natl Acad Sci USA 93:8016-8021.

Canli T, Zhao Z, Brewer J, Gabrieli JD, Cahill L (2000) Event-related activation in the human amygdala associates with later memory for individual emotional experience. J Neurosci 20:RC99(1-5).

Capitani E, Della Sala S, Logie RH, Spinnler H (1992) Recency, primacy, and memory: reappraising and standardising the serial position curve. Cortex 28:315-342.

Christianson SA (1984) The relationship between induced emotional arousal and amnesia. Scand J Psychol 25:147-160.

Cycowicz YM, Friedman D, Rothstein M, Snodgrass JG (1997) Picture naming by young children: norms for name agreement, familiarity, and visual complexity. J Exp Child Psychol 65:171-237.

Dolan RJ (2002) Emotion, cognition, and behavior. Science 298:1191-1194.

Dolcos F, LaBar KS, Cabeza R (2004a) Interaction between the amygdala and the medial temporal lobe memory system predicts better memory for emotional events. Neuron 42:855-863.

Dolcos F, LaBar KS, Cabeza R (2004b) Dissociable effects of arousal and valence on prefrontal activity indexing emotional evaluation and subsequent memory: an event-related fMRI study. NeuroImage 23:64-74.

Easterbrook JA (1959) The effect of emotion on cue utilization and the organization of behavior. Psychol Rev 66:183-201.

Frahnert C, Rao ML, Grasmaeder K (2003) Determination of eighteen antidepressants, four antipsychotics and active metabolites in serum by liquid chromatography: a simple tool for therapeutic drug monitoring. J Chromatogr B Biomed Sci Appl 794:35-47.

Gold PE, van Buskirk R (1978) Posttraining brain norepinephrine concentrations: correlation with retention performance of avoidance training and with peripheral epinephrine modulation of memory processing. Behav Biol 23:509-520.

Hamann S (2001) Cognitive and neural mechanisms of emotional memory. Trends Cogn Sci 5:394-400.

Hamann SB, Ely TD, Grafton ST, Kilts CD (1999) Amygdala activity related to enhanced memory for pleasant and aversive stimuli. Nat Neurosci 2:289-293

Harmer CJ, Hill SA, Taylor MJ, Cowen PJ, Goodwin GM (2003) Toward a neuropsychological theory of antidepressant drug action: increase in positive emotional bias after potentiation of norepinephrine activity. Am J Psychiatry 160:990-992.

Harmer CJ, Shelley NC, Cowen PJ, Goodwin GM (2004) Increased positive versus negative affective perception and memory in healthy volunteers following selective serotonin and norepinephrine reuptake inhibition. Am J Psychiatry 161:1256-1263.

Kensinger EA (2004) Remembering emotional experiences: the contribution of valence and arousal. Rev Neurosci 15:241-251.

Kensinger EA, Corkin S (2004) Two routes to emotional memory: distinct neural processes for valence and arousal. Proc Natl Acad Sci USA 101:3310-3315.

Lang PJ, Bradley MM, Cuthberg BN (1997) International Affective Picture System. Gainesville, FL: NIMH Center for the Study of Emotion and Attention.

Loftus EF (1979) The malleability of human memory. Am Sci 67:312-320.

Loftus EF, Burns TE (1982) Mental shock can produce retrograde amnesia. Mem Cognit 10:318-323.

McGaugh JL (2000) Memory-a century of consolidation. Science 287:248-251.

McGaugh JL, Roozendaal B (2002) Role of adrenal stress hormones in forming lasting memories in the brain. Curr Opin Neurobiol 12:205-210.

McGinty D, Szymusiak R (1988) Neuronal unit activity patterns in behaving animals: brainstem and limbic system. Annu Rev Psychol 39:135-168.

Murchison CF, Zhang XY, Zhang WP, Ouyang M, Lee A, Thomas SA (2004) A distinct role for norepinephrine in memory retrieval. Cell 117:131-143.
O'Carroll RE, Drysdale E, Cahill L, Shajahan P, Ebmeier KP (1999a) Stimulation of the noradrenergic system enhances and blockade reduces memory for emotional material in man. Psychol Med 29:1083-1088.

O'Carroll RE, Drysdale E, Cahill L, Shajahan P, Ebmeier KP (1999b) Memory for emotional material: a comparison of central versus peripheral beta blockade. J Psychopharmacol 13:32-39.

Papps BP, Shajahan PM, Ebmeier KP, O'Carroll RE (2002) The effects of noradrenergic re-uptake inhibition on memory encoding in man. Psychopharmacology 159:311-318.

Phelps EA (2004) Human emotion and memory: interactions of the amygdala and hippocampal complex. Curr Opin Neurobiol 14:198-202.

Pitkanen A, Pikkarainen M, Nurminen N, Ylinen A (2000) Reciprocal connections between the amygdala and the hippocampal formation, perirhinal cortex, and postrhinal cortex in rat. A review. Ann NY Acad Sci 911:369-391.

Raymond JE, Shapiro KL, Arnell KM (1992) Temporary suppression of visual processing in an RSVP task: an attentional blink? J Exp Psychol Hum Percept Perform 18:849-860.

Richardson MP, Strange BA, Dolan RJ (2004) Encoding of emotional memories depends on amygdala and hippocampus and their interactions. Nat Neurosci 7:278-285.

Sara SJ, Vankov A, Hervé A (1994) Locus coeruleus-evoked responses in behaving rats: a clue to the role of noradrenaline in memory. Brain Res Bull 35:457-465.

Small DM, Gregory MD, Mak YE, Gitelman D, Mesulam MM, Parrish T (2003) Dissociation of neural representation of intensity and affective valuation in human gustation. Neuron 39:701-711.

Snodgrass JG, Vanderwart M (1980) A standardized set of 260 pictures: norms for name agreement, image agreement, familiarity, and visual complexity. J Exp Psychol [Hum Learn] 6:174-215.

Southwick SM, Davis M, Horner B, Cahill L, Morgan III CA, Gold PE, Bremner JD, Charney DC (2002) Relationship of enhanced norepinephrine activity during memory consolidation to enhanced long-term memory in humans. Am J Psychiatry 159:1420-1422.

Sternberg DB, Gold PE (1981) Retrograde amnesia produced by electrical stimulation of the amygdala: attenuation with adrenergic antagonists. Brain Res 211:59-65.

Strange BA, Dolan RJ (2004) Beta-adrenergic modulation of emotional memory-evoked human amygdala and hippocampal responses. Proc Natl Acad Sci USA 101:11454-11458.

Strange BA, Hurlemann R, Dolan RJ (2003) An emotion-induced retrograde amnesia in humans is amygdala- and beta-adrenergic-dependent. Proc Natl Acad Sci USA 100:13626-13631.

Tulving E (1969) Retrograde amnesia in free recall. Science 164:88-90.

Tulving E, Markowitsch HJ, Craik FE, Habib R, Houle S (1996) Novelty and familiarity activations in PET studies of memory encoding and retrieval. Cereb Cortex 6:71-79.

van Stegeren AH, Everaerd W, Cahill L, McGaugh JL, Gooren LJ (1998) Memory for emotional events: differential effects of centrally versus peripherally acting beta-blocking agents. Psychopharmacology 138:305-310.

van Stegeren AH, Goekoop R, Everaerd W, Scheltens P, Barkhof F, Kuijer JP, Rombouts SA (2005) Noradrenaline mediates amygdala activation in men and women during encoding of emotional material. NeuroImage 24:898-909.

von Restorff H (1933) Ueber die Wirkungen von Bereichsbildung im Spurenfeld. Psychol Forsch 18:299-342.

Wallace WP (1965) Review of the historical empirical and theoretical status of the von Restorff phenomenon. Psychol Bull 63:410-424.

Wise RA (2004) Dopamine, learning and motivation. Nat Rev Neurosci 5:483-494.

Yamasaki H, LaBar KS, McCarthy G (2002) Dissociable prefrontal brain systems for attention and emotion. Proc Natl Acad Sci USA 99:1144711451. 